\title{
ORIGINAL ARTICLE \\ Development of a modified model of spinal cord ischemia injury by selective ligation of lumbar arteries in rabbits
}

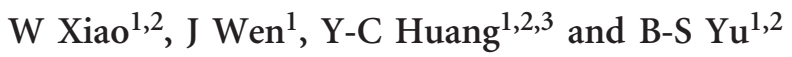

\author{
Study design: Experimental study. \\ Objective: The aim of this study is to develop a modified model of spinal cord ischemia in rabbits. \\ Settings: Shenzhen Key Laboratory of Spine Surgery, Shenzhen, China.
}

Methods: In total, $20 \mathrm{New}$ Zealand rabbits were divided into the following four groups according to the level of ligation of bilateral lumbar arteries: (1) group A, sham group, no ligation, $n=5$; (2) group B, ligation of bilateral lumbar arteries at three levels (L2-L4, $n=5)$; (3) group C, ligation of bilateral lumbar arteries at four levels (L2-L5, $n=5)$; and (4) group D, ligation of bilateral lumbar arteries at five levels ( $L 1-L 5, n=5$ ). The latency of motor-evoked potentials was measured intraoperatively and the modified Tarlov grades were scored, followed by a histological observation of spinal cord, on the seventh day after surgery.

Results: All 10 rabbits in Group A and Group B were electrophysiologically, neurologically and histologically normal. In Group C, moderate spinal cord ischemia injury was found in three of five rabbits: they had prolonged latency of motor-evoked potentials and neuronal karyopyknosis in the anterior horn of spinal cord, and the average Tarlov score was $4.2 \pm 0.8$. In Group D, severe spinal cord ischemia injury was recorded in all the five rabbits: the latency of motor-evoked potential prolonged in one rabbit, whereas the waveform disappeared in four rabbits; loss of neurons and vacuolation of gray matter were seen in spinal cord sections, and the average Tarlov score was $0.6 \pm 0.9$.

Conclusion: Selective ligation of lumbar arteries was a modified method to induce feasible and reproducible model of spinal cord ischemia in rabbits.

Spinal Cord (2017) 55, 1028-1032; doi:10.1038/sc.2017.66; published online 13 June 2017

\section{INTRODUCTION}

Paraplegia caused by spinal cord ischemia remains a devastating and unpredictable complication of operations in which interruption of aorta or segmental arteries is required, such as aortic repair surgery ${ }^{1}$ and multilevel total en bloc spondylectomy. ${ }^{2,3}$ Although great improvements in the prevention and treatment of spinal cord ischemia have been achieved, this complication still affects the quality of life severely. Spinal cord ischemia injury is of great interest in the field of neurology, and a reliable and reproducible experimental model is crucial for the basic scientists and the surgeons to understand the pathophysiology and to develop clinical therapeutic strategies effectively.

To date, many animal models of spinal cord ischemia have been developed, and most of them are induced by cross-clamping the thoracic or abdominal aorta temporarily or irreversibly. ${ }^{4-7}$ These models have significant merit, but critical limitations remain. Irreversible occlusion of the aorta to induce spinal cord ischemia, with resultant shortage of blood perfusion distal to the clamping site, such as ischemia of lower limbs and dysfunction of abdominal organs, may affect the evaluation of neurological function and analysis of the treatment effect. ${ }^{8}$ Hence, a method to induce spinal cord ischemia and to keep the intact blood flow in aorta in animals is needed.
To develop this new method for spinal cord ischemia injury, the rabbits were used in this study because of the similar vascular anatomy of spinal cord with that of humans. ${ }^{9,10}$ Ligating the lumbar arteries in rabbits was used to develop spinal cord ischemia and to ensure the intact blood flow in the aorta. The influence of ligation of lumbar arteries on the structure and function of spinal cord was then assessed electrophysiologically, neurologically and histologically to test the success of this model.

\section{MATERIALS AND METHODS}

All experimental protocols of this study were approved by the experimental animal welfare ethics committee of Shenzhen Peking University-Hong Kong University of Science and Technology Medical Center. The experimental animals were obtained from the Guangdong Medical Laboratory Animal Center, and they were housed individually in metal cages and provided with food and water. A total of 20 New Zealand rabbits (age 3 months, weight $1.8-2.5 \mathrm{~kg}$, average weight $2.2 \mathrm{~kg}$ ) were used. The animals were divided into four groups as follows: group A, sham group, no ligation, $n=5$; group $\mathrm{B}$, ligation of bilateral lumbar arteries at three levels (L2-L4, $n=5$ ); group C, ligation of bilateral lumbar arteries at four levels (L2-L5, $n=5$ ); and group D, ligation of bilateral lumbar arteries at five levels (L1-L5, $n=5)$.

${ }^{1}$ Shenzhen Key Laboratory of Spine Surgery, Department of Spine Surgery, Peking University Shenzhen Hospital, Shenzhen, China; ${ }^{2}$ Shenzhen Engineering Laboratory of Orthopaedic Regenerative Technologies, Orthopaedic Research Center, Peking University Shenzhen Hospital, Shenzhen, China and ${ }^{3}$ Beijing Key Laboratory for Genetic Research of Bone and Joint Disease, Central Laboratory, Peking Union Medical College Hospital, Peking Union Medical College and Chinese Academy of Medical Sciences, Beijing, China Correspondence: Professor B-S Yu, Shenzhen Key Laboratory of Spine Surgery, Department of Spine Surgery, Peking University Shenzhen Hospital, No. 1120, Lianhua Road, Shenzhen 518036, China.

E-mail: doctorxiaowei@icloud.com or hpyubinsheng@hotmail.com

Received 1 December 2016; revised 30 April 2017; accepted 4 May 2017; published online 13 June 2017 

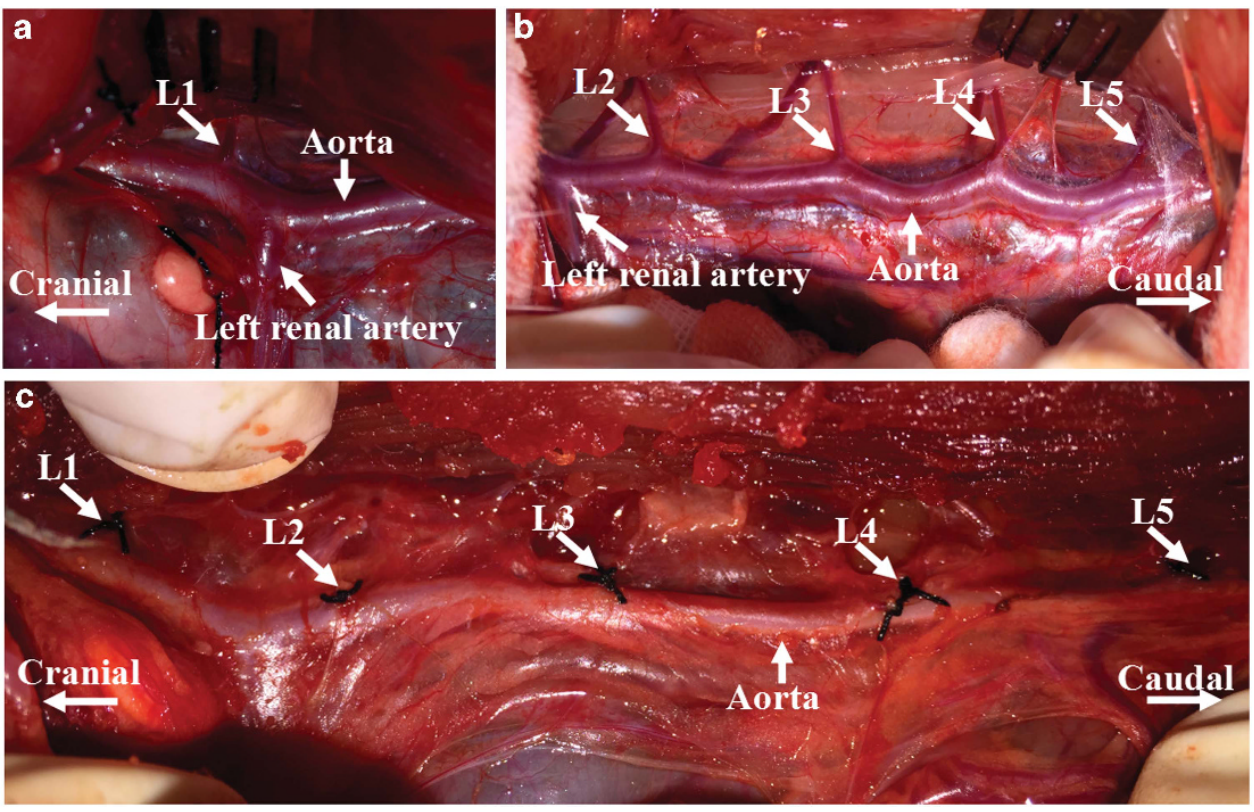

Figure 1 Exposure and anatomy of the aorta and lumbar arteries. L1 artery was cranial to the left renal artery (a); L2-L5 arteries were distal to the left renal artery (b); lumbar arteries were ligated and cut off from L1 to L5 (c).

\section{Table 1 Modified Tarlov grading system}

\begin{tabular}{ll}
\hline Grade 0 & Complete paraplegia with no hind extremity function \\
Grade 1 & Minor joint movements \\
Grade 2 & Major joint movements \\
Grade 3 & Animal can stand \\
Grade 4 & Animal can walk \\
Grade 5 & Animal can climb a $20^{\circ}$ inclined plane
\end{tabular}

\section{Surgical procedure}

The rabbits were fasted overnight for at least $8 \mathrm{~h}$ before operation, after being anesthetized with pentobarbital sodium $\left(35 \mathrm{mg} \mathrm{kg}^{-1}\right.$; Sigma, St Louis, MO, USA) by the ear vein injection; they were placed on an operating table with a heat lamp to maintain the body temperature at about $37^{\circ} \mathrm{C}$. The center ear artery was chosen for invasive blood pressure monitoring; after ligation of the center ear artery, a catheter was placed into the artery proximally. The catheter was filled with $1 \%$ heparin solution, and then a transducer was connected to the catheter, which was able to measure the blood pressure and transfer pressure signal into electric signal. Thus, the real-time blood pressure was presented as a continuous curve on computer screen (MP150, BIOPAC, Goleta, CA, USA). Painful stimulation should not cause any increase of blood pressure or pain reflex during anesthesia. All the rabbits were placed in the right lateral decubitus position to make a left paramedian incision. The abdominal aorta and lumbar arteries were exposed through a retroperitoneal approach. The New Zealand rabbit has six pair of lumbar arteries in total, ${ }^{9}$ most of these arteries originate from a common trunk on aorta. ${ }^{10}$ The left renal artery acts as an anatomic marker for recognizing the level of lumbar arteries: L1 artery was cranial to the left renal artery (Figure 1a), and arteries of L2-L5 were distal to it (Figure 1b). The bilateral lumbar arteries were first ligated with silk at a different level according to different groups, they were then cut off to make sure the segmental blood supply to spinal cord was interrupted completely (Figure 1c). Intramuscular injection of Penicillin was used intraoperatively and postoperatively to prevent wound infection.

\section{Electrophysiological testing}

The neural conductive function of spinal cord in rabbits was evaluated by motor-evoked potentials produced by electrical stimulation (Cascade, Cadwell, Kennewick, WA, USA). ${ }^{11,12}$ Two stimulating needle electrodes were placed on bilateral scalp at $1 \mathrm{~cm}$ cranial to the root of ears, and the compound muscle action potentials were recorded from the bilateral anterior-thigh muscles with needle electrodes. The stimulation signal used a rectangular waveform with the following parameters: a pulse duration of 0.5 millisecond, an interstimulus interval of 2.0 millisecond and a train of four pulses per stimulation at $1 \mathrm{~Hz}$. The stimulation intensity, which ranged from 60 to $120 \mathrm{~V}$, was set to $10 \%$ above the level that elicited the maximal amplitude. The band pass filter was set at $10-3000 \mathrm{~Hz}$. Motor-evoked potentials were recorded before ligation, at $10 \mathrm{~min}$ and at $1 \mathrm{~h}$ after ligation. Considering the amplitude of MEP was not stable enough and could be influenced by anesthetic drugs, ${ }^{13}$ the latency was used as a reliable indicator for evaluation of the neural conductive function of spinal cord. ${ }^{14}$ The latency of the first negative peak was measured, and the one that prolonged more than $10 \%$ of the baseline was considered to be abnormal. ${ }^{15}$

\section{Neurological assessment}

Postoperative neurological function was assessed with a modified Tarlov grading system on the seventh day after ligation of bilateral segmental arteries. ${ }^{16}$ Grades were assigned as follows: 0 , complete paraplegia with no hind extremity motion; 1, minor joint movements; 2, major joint movements; 3 , the animals can stand; 4 , the animals can walk; and 5 , the animals can climb a $20^{\circ}$ inclined plane (Table 1).

\section{Histological examination}

After neurological function assessment, the animal was anesthetized and killed using an overdose injection of pentobarbital sodium through the ear vein. Then the lumbar spinal column (from L1 to L5 vertebrae) together with paravertebral muscles were quickly harvested and immersed into neutral buffered $10 \%$ formalin solution. After being fixed in formalin solution for 5 days, the lumbar spinal cord was dissected out and immersed in 50, 70, 80, 90 and $95 \%$ concentrations of alcoholic solutions consecutively for dehydration (lasting for $40 \mathrm{~min}$ in each concentration). The spinal cord was then cut out at L3/4 level and embedded in paraffin. The sections at $5 \mu \mathrm{M}$ were stained with hematoxylineosin and Nissl's staining. The microstructures in the anterior horn of the spinal cord were observed at the magnification of $\times 40, \times 100$ and $\times 400$.

\section{Statistical analysis}

Data were given as mean \pm standard deviation, the results of arterial pressure were analyzed by analysis of variance followed by a least significant difference test. 
Table 2 Blood pressure analysis before and after ligation of lumbar arteries

\begin{tabular}{lcc}
\hline \multirow{2}{*}{ Group } & \multicolumn{2}{c}{ Blood pressure $(\mathrm{mm} \mathrm{Hg})$} \\
\cline { 2 - 3 } & Baseline & After ligation \\
\hline A & $80.4 \pm 1.5$ & \\
B & $78.5 \pm 3.6$ & $77.4 \pm 4.0$ \\
C & $81.6 \pm 2.0$ & $80.5 \pm 3.3$ \\
D & $78.0 \pm 2.5$ & $79.8 \pm 1.4$ \\
\hline
\end{tabular}

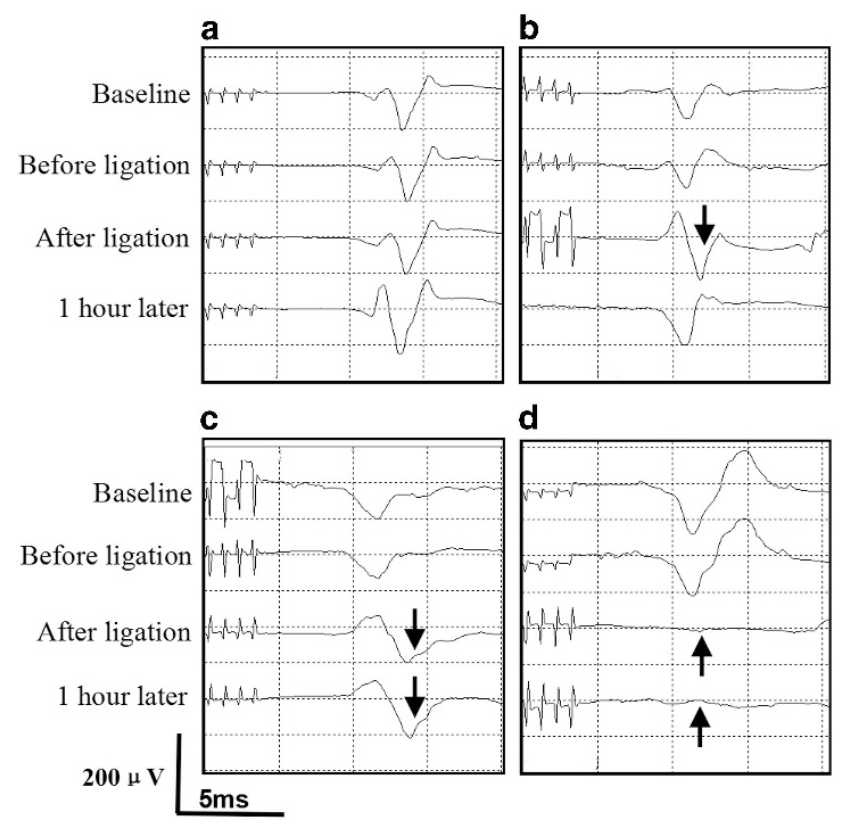

Figure 2 Samples for four types of motor-evoked potentials changing with time. Latency remained normal (a); latency prolonged transiently (b); latency prolonged continually (c) and waveform disappeared after ligation (d). The arrows indicated for the prolonged latency or disappearance of the first negative peak.

And the analysis of Tarlov score was performed with Kruskal-Wallis rank-sum test. Difference with $P$-values less than 0.05 was considered statistically significant. All the analyses were performed using SPSS 18.0 (IBM, Armonk, NY, USA).

\section{RESULTS}

Lumbar arteries were successfully ligated via the retroperitoneal approach

No significant difference was found in mean arterial blood pressure among the four groups during the baseline period; in addition, ligation of lumbar arteries did not change the mean arterial blood pressure significantly in any group (Table 2). Except for five sham-operated rabbits in group A, lumbar arteries were successfully ligated at three to five levels in 15 of 18 rabbits; three rabbits died of massive bleeding from the aorta because the lumbar arteries were torn during the operation. Hence, totally 60 arteries were successfully ligated among 63 , namely a $95 \%$ success rate of lumbar artery ligation in this experiment.

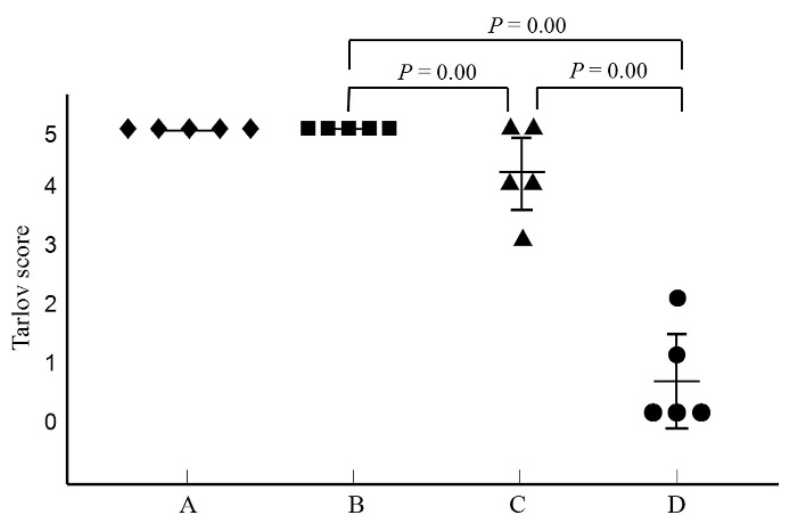

Figure 3 Tarlov score of the rabbits at the seventh day after the operation in four groups. All the rabbits in Group A and Group B, and two rabbits in Group C were neurologically normal. Motor dysfunction developed in three rabbits in Group C and two rabbits in Group D. Complete paraplegia developed in three rabbits in Group D.

\section{Ligation of lumbar arteries at $\geqslant 4$ levels induced spinal cord ischemia injury}

The latency of motor-evoked potential showed no abnormal change in Group A and Group B (Figure 2a). All 10 rabbits in these two groups were neurologically normal after operation (modified Tarlov grade 5, Figure 3), and no sign of neurohistological damage was observed in the spinal cord after histological staining (Figure 4). Thus ligation of lumbar arteries at three levels was safe for the spinal cord in rabbits.

In Group C, the latency of motor-evoked potentials remained normal in two rabbits after operation. In the other three rabbits, the latency prolonged more than $10 \%$ of baseline at $10 \mathrm{~min}$ after ligation and it recovered to be normal in two of them $1 \mathrm{~h}$ later (Figure $2 \mathrm{~b}$ ), but this recovery was not found in the other rabbit (Figure 2c). The average Tarlov score in this group was $4.2 \pm 0.8$ (Figure 3). The cell volume of neuron decreased obviously and karyopyknosis was observed in the stained sections of the spinal cord, whereas the colors of both white matter and gray matter were normally stained in hematoxylin-eosin and Nissl's staining (Figure 4). The results demonstrated that moderate spinal cord ischemia injury was formed in these rabbits.

In Group $\mathrm{D}$, the latency of motor-evoked potentials prolonged more than $10 \%$ of baseline in one rabbit after ligation, which remained so $1 \mathrm{~h}$ later (Figure $2 \mathrm{c}$ ). The waveform of motor-evoked potentials disappeared in the other four rabbits after ligation by $1 \mathrm{~h}$ (Figure 2d). In this group, the average Tarlov score was $0.6 \pm 0.9$ (Figure 3). Loss of neuron and vacuolation of gray matter were observed in the stained sections of spinal cord, and the colors of both white matter and gray matter were obviously light in hematoxylineosin and Nissl's staining (Figure 4). The results determined that severe spinal cord ischemia injury was developed in this group.

\section{DISCUSSION}

Occlusion of the lumbar arteries is not a novel method for the induction of spinal cord ischemia, as Maeda et al. ${ }^{8}$ have reported to induce spinal cord ischemia in rabbits by temporary occlusion of five lumbar arteries and they found the protective effect of systemic mild hypothermia. Nevertheless, in the present study, a spinal cord ischemia model was established by irreversible selective ligation of lumbar arteries, and we clearly described this model using the results of electrophysiological, neurological and histological assessments; moreover, we found that moderate and severe ischemia injury of 


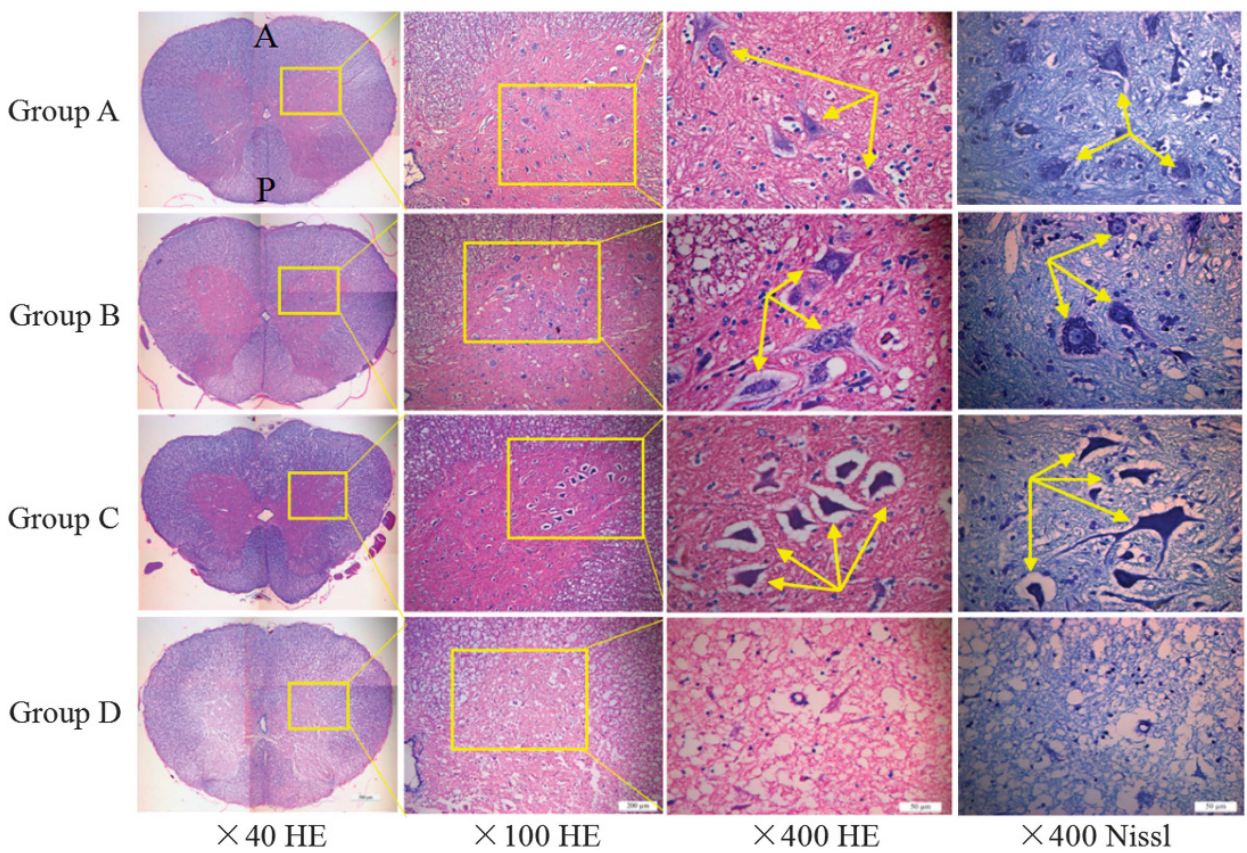

Figure 4 HE (hematoxylin-eosin) and Nissl's staining of spinal cord sections at the seventh day after operation. No sign of neuronal damage was observed in Group A and Group B; decrease of the cell volume of neuron and karyopyknosis were observed in Group C; reduction of the number of neuron and vacuolation of gray matter were observed in Group D. The frames indicated the anterior horns and the arrows indicated the neurons.

spinal cord can be successfully induced by ligating lumbar arteries at four and five levels, respectively.

In addition to the anterior and posterior spinal arteries, the spinal cord is nourished by segmental arteries ${ }^{17}$ that originate from aorta and give off branches joining in the anterior and posterior spinal arteries. ${ }^{18}$ Anatomically, occlusion of the aorta causes a decrease of spinal cord blood flow and thus increases the risk of spinal cord ischemia injury. ${ }^{19}$ Most of the currently experimental models of spinal cord ischemia were achieved by clamping the aorta transiently or constantly. ${ }^{4-7}$ In these models, not only the blood flow in the segmental arteries but also the blood supply to lower limbs and to abdominal organs was interrupted. Thus, the motor dysfunction of lower limbs and sphincters may be owing to ischemia of both the spinal cord and the musculature, which may influence the accuracy of neurological assessment in experiments. ${ }^{8}$

In this study, a rabbit model of spinal cord ischemia was developed by ligation of lumbar arteries without interrupting the blood flow in aorta. Thus, one advantage of this model is that the lower limbs and abdominal organs were prevented from ischemia injury, and the hemodynamic changes of animals were minimal as all the rabbits had steady blood pressure during physiological and ischemic period; the results of neurological assessments thus should be much more objective because the potential influence from lower limbs' ischemia was eliminated. Another advantage of this model was that the different extent of spinal cord ischemia injury could be easily produced by ligation of lumbar arteries at different levels.

In this work, it was found that ligation of lumbar arteries at four levels was capable of producing spinal cord ischemia. Fujimaki and Ueda found that ligation of segmental arteries at $\leqslant 4$ levels was safe, but the spinal cord blood flow was reduced to $70 \%$ of the normal value in dogs; ${ }^{20,21}$ if the ligation of segmental arteries was at $\geqslant 5$ levels, spinal cord ischemia was found and the spinal cord blood flow was reduced to $40-50 \%$ of the normal value. ${ }^{21}$ This finding pointed out that the number of ligation of segmental arteries for spinal cord ischemia is variable in different animal species; possibly there is a critical threshold level of blood perfusion to ensure the neural function of spinal cord..$^{21}$

Multilevel total en bloc spondylectomy requires ligation of several pairs of bilateral segmental arteries, which always leads to the surgeon's concern about postoperative complications related to spinal cord ischemia. Murakami et al. ${ }^{22}$ suggested that during multilevel total en bloc spondylectomy, the surgeons are allowed to kill up to three pairs of segmental arteries, even including the Adamkiewicz artery. But the formation of ischemic spinal cord dysfunction caused by ligations of segmental arteries remains unpredictable. For instance, Luzzati et $a .^{2}$ reported that three in nine patients developed paraplegia after four to five levels of total en bloc spondylectomy, and 1 in 20 patients complicated with spinal cord ischemia injury after two to five levels of total en bloc spondylectomy was reported by Disch et al. ${ }^{3}$ Hence, this rabbit is more suitable to investigate the influence of ligation of segmental arteries on the spinal cord, because it is able to mimic the clinical intraoperative situations of multilevel total en bloc spondylectomy. The ligation of segmental arteries at different levels provided a model of consecutive decrease of spinal cord blood flow, ${ }^{20,21}$ which enabled researchers to observe the dynamic progress of spinal cord ischemia. Hence, this model was very meaningful to study the pathophysiology during the development of spinal cord ischemia injury.

However, there are several limitations we should acknowledge in this study. First, the somatosensory-evoked potentials were not measured in these rabbits; hence how the ligation of arteries affected the sensory conductive function of spinal cord was unclear. In addition, the real-time change of spinal cord blood perfusion was not obtained intraoperatively because of the lack of direct monitoring of spinal cord blood flow. Even though electrophysiological monitoring of spinal cord has been proved to be sensitive and effective to see intraoperative spinal cord injury, ${ }^{23-25}$ whether electrophysiological signal is capable of monitoring the real-time spinal cord blood 
flow remains unclear. ${ }^{26,27}$ Therefore, the correlation between electrophysiological monitoring and spinal cord blood flow required further investigations. Third, the results from this animal model cannot be easily extrapolated to humans as the anatomical difference; but these findings are consistent with previous knowledge in humans and may provide useful tips to evaluate the safety of ligation of bilateral segmental arteries.

\section{CONCLUSION}

The ligation of bilateral lumbar arteries at $>3$ levels has significant impairment on the structure and function of spinal cord in rabbits, which can be used as a modified method to establish a feasible and reproducible model of spinal cord ischemia. Compared with cross-clamping the aorta, ligation of lumbar artery has less impact on the hemodynamics as the blood flow in aorta is well kept. Furthermore, this model can produce moderate and severe ischemia injury of spinal cord by ligating lumbar arteries at 4 and 5 levels, respectively.

\section{DATA ARCHIVING}

There were no data to deposit.

\section{CONFLICT OF INTEREST}

The authors declare no conflict of interest.

\section{ACKNOWLEDGEMENTS}

This study was financially supported by National Natural Science Foundation of China (No.U1613224) and Shenzhen Science and Technology Innovation Committee Projects (Nos. JCYJ20130402113615042 \& JCYJ20150403091443318).

1 Yamauchi T, Takano H, Nishimura M, Matsumiya G, Sawa Y. Paraplegia and paraparesis after descending thoracic aortic aneurysm repair: a risk factor analysis. Ann Thorac Cardiovasc Surg 2006; 12: 179-183.

2 Luzzati AD, Shah SP, Gagliano FS, Perrucchini GG, Fontanella W, Alloisio M. Four- and five- level en bloc spondylectomy for malignant spinal tumors. Spine (Phila Pa 1976) 2014; 39: E129-E139.

3 Disch AC, Schaser KD, Melcher I, Feraboli F, Schmoelz W, Druschel C et al. Oncosurgical results of multilevel thoracolumbar en-bloc spondylectomy and reconstruction with a carbon composite vertebral body replacement system. Spine (Phila Pa 1976) 2011; 36: E647-E655.

4 Gurer B, Kertmen H, Kasim E, Yilmaz ER, Kanat BH, Sargon MF et al. Neuroprotective effects of testosterone on ischemia/reperfusion injury of the rabbit spinal cord. Injury 2015; 46: 240-248.

5 Song W, Sun J, Su B, Yang R, Dong H, Xiong L. Ischemic postconditioning protects the spinal cord from ischemia-reperfusion injury via modulation of redox signaling. J Thorac Cardiovasc Surg 2013; 146: 688-695.

6 Basoglu H, Kurtoglu T, Cetin NK, Bilgin MD, Kiylioglu N. Assessment of in vivo spinal cord conduction velocity in rats in an experimental model of ischemic spinal cord injury. Spinal Cord 2013; 51: 616-622.
7 Izumi S, Okada K, Hasegawa T, Omura A, Munakata H, Matsumori M et al. Augmentation of systemic blood pressure during spinal cord ischemia to prevent postoperative paraplegia after aortic surgery in a rabbit model. J Thorac Cardiovasc Surg 2010; 139: 1261-1268.

8 Maeda T, Mori K, Shiraishi Y, Tatebayashi K, Kawai Y. Selective occlusion of lumbar arteries as a spinal cord ischemia model in rabbits. Jpn J Physiol 2003; 53: 9-15.

9 Mazensky D, Radonak J, Danko J, Petrovova E, Frankovicova M. Anatomical study of blood supply to the spinal cord in the rabbit. Spinal Cord 2011; 49: 525-528.

10 Mazensky D, Danko J, Petrovova E, Mechirova E, Prokes M. Arterial peculiarities of the thoracolumbar spinal cord in rabbit. Anat Histol Embryol 2014; 43: 346-351.

11 Nakanishi K, Tanaka N, Fujiwara Y, Kamei N, Ochi M. Corticospinal tract conduction block results in the prolongation of central motor conduction time in compressive cervical myelopathy. Clin Neurophysiol 2006; 117: 623-627.

12 Kaneko K, Taguchi T, Morita H, Yonemura H, Fujimoto H, Kawai S. Mechanism of prolonged central motor conduction time in compressive cervical myelopathy. Clin Neurophysiol 2001; 112: 1035-1040.

13 Lo YL, Dan YF, Tan YE, Nurjannah S, Tan SB, Tan CT et al. Intraoperative motor-evoked potential monitoring in scoliosis surgery: comparison of desflurane/nitrous oxide with propofol total intravenous anesthetic regimens. J Neurosurg Anesthesiol 2006; 18: 211-214.

14 Malhotra NR, Shaffrey Cl. Intraoperative electrophysiological monitoring in spine surgery. Spine (Phila Pa 1976) 2010; 35: 2167-2179.

15 Kobayashi S, Matsuyama Y, Shinomiya K, Kawabata S, Ando M, Kanchiku T et al. A new alarm point of transcranial electrical stimulation motor evoked potentials for intraoperative spinal cord monitoring: a prospective multicenter study from the Spinal Cord Monitoring Working Group of the Japanese Society for Spine Surgery and Related Research. J Neurosurg Spine 2014; 20: 102-107.

16 Tarlov IM, Klinger H. Spinal cord compression studies. II. Time limits for recovery after acute compression in dogs. AMA Arch Neurol Psychiatry 1954; 71: 271-290.

17 Melissano G, Bertoglio L, Rinaldi E, Leopardi M, Chiesa R. An anatomical review of spinal cord blood supply. J Cardiovasc Surg (Torino) 2015; 56: 699-706.

18 Colman MW, Hornicek FJ, Schwab JH. Spinal cord blood supply and its surgical implications. J Am Acad Orthop Surg 2015; 23: 581-591.

19 Etz DC, Luehr M, Aspern KV, Misfeld M, Gudehus S, Ender J et al. Spinal cord ischemia in open and endovascular thoracoabdominal aortic aneurysm repair: new concepts. $J$ Cardiovasc Surg (Torino) 2014; 55: 159-168.

20 Ueda Y, Kawahara N, Tomita K, Kobayashi T, Murakami H, Nambu K. Influence on spinal cord blood flow and function by interruption of bilateral segmental arteries at up to three levels: experimental study in dogs. Spine 2005; 30: 2239-2243.

21 Fujimaki Y, Kawahara N, Tomita K, Murakami H, Ueda Y. How many ligations of bilateral segmental arteries cause ischemic spinal cord dysfunction? An experimental study using a dog model. Spine (Phila Pa 1976) 2006; 31: E781-E789.

22 Murakami H, Kawahara N, Tomita K, Demura S, Kato S, Yoshioka K. Does interruption of the artery of Adamkiewicz during total en bloc spondylectomy affect neurologic function? Spine (Phila Pa 1976) 2010; 35: E1187-E1192.

23 Tamaki T, Kubota S. History of the development of intraoperative spinal cord monitoring. Eur Spine J 2007; 16 (Suppl 2): 140-146.

24 Wen CY, Cui JL, Mak KC, Luk KD, Hu Y. Diffusion tensor imaging of somatosensory tract in cervical spondylotic myelopathy and its link with electrophysiological evaluation. Spine J 2014; 14: 1493-1500.

25 Owen $\mathrm{JH}$. The application of intraoperative monitoring during surgery for spinal deformity. Spine (Phila Pa 1976) 1999; 24: 2649-2662.

26 Elmore JR, Gloviczki P, Harper CM, Pairolero PC, Murray MJ, Bourchier RG et al. Failure of motor evoked potentials to predict neurologic outcome in experimental thoracic aortic occlusion. J Vasc Surg 1991; 14: 131-139.

27 Hitchon PW, Dyste GN, Osenbach RK, Jensen AE. Response of spinal cord blood flow and motor and sensory evoked potentials to aortic ligation. Surg Neurol 1990; 34: 279-285. 